\title{
Impacto en los niveles de deserción y reprobación en los estudiantes usuarios de la estancia infantil de la Universidad Tecnológica de Chihuahua
}

\section{Dropout and impact failure of students users of the childrens stay in at the Universidad Tecnológica de Chihuahua}

\author{
ANCHONDO-CHAVARRIA, Guadalupe*†, MARTÍNEZ-ARANDA, Rebeca y PRIETO-GARCÍA, \\ Brenda
}

Universidad Tecnológica de Chihuahua

ID $1^{\text {er }}$ Autor: Guadalupe, Anchondo-Chavarría / ORC ID: 0000-0002-2282-0822, CVU CONACYT ID: 520858

ID 1 ${ }^{\text {er }}$ Coautor: María Rebeca, Martínez-Aranda / ORC ID: 0000-0001-6012-2905

ID $2^{\text {do }}$ Coautor: Brenda, Prieto-García / ORC ID: 0000-0002-0168-338X

DOI: $10.35429 / J U M .2019 .7 .3 .9 .15$

Recibido 10 de Enero, 2019; Aceptado 30 de Marzo, 2019

\begin{abstract}
Resumen
Ser padre o madre de familia y estudiante al mismo tiempo, es un reto cada vez más frecuente entre los jóvenes en la ciudad de Chihuahua. La Universidad Tecnológica al contar con un importante número de alumnos en esta situación y con dificultad para el cuidado de sus hijos mientras asisten a clases, decide abrir una estancia infantil, con un horario compatible con sus estudios. El proyecto tiene como objetivo medir el impacto que tiene la estancia en los índices de reprobación, permanencia y deserción, en los usuarios del servicio a tres años de su fundación. Se utiliza el tipo de investigación concluyente, descriptiva y transversal, a través de un cuestionario estructurado y análisis de estadísticas de los estudiantes que utilizan el servicio. Los resultados más relevantes son: $82.6 \%$ son mujeres madres de familia, la mayoría utiliza el servicio desde que iniciaron en la universidad y el $87 \%$ decidió entrar a la UTCH por contar con una estancia infantil, el 91\% asegura que ha disminuido el índice de reprobación y mejorado su aprovechamiento académico. Los estudiantes perciben que la estancia les ha brindado beneficios económicos, académicos y seguridad al tener a sus hijos cerca y atendidos por personal experto.
\end{abstract}

Estancia infantil, Índice de permanencia y deserción

\begin{abstract}
Being a father and mother and student at the same time is an increasingly frequent challenge among young people in the city of Chihuahua. The Technological University, having an important number of students in this situation and with the difficulty to looking after their children while attending classes, decides to open a day-care center, with a compatible schedule with their studies. The project aims to measure the impact that the nursery has on the rates of failure, permanence and attrition, with the users of the service three years after its foundation. The type of conclusive, descriptive and cross-sectional research is used through a structured questionnaire and statistics analysis of the students who use the service. The most relevant results are: $82.6 \%$ are women mothers, most of them use the service since they started in college and $87 \%$ decided to enter the UTCH for having a day-care center, and $91 \%$ said the failure index has decreased and the academic achievement has improved. Students perceive that the day-care has provided them with economic, academic and security benefits by having their children close and attended by expert staff.
\end{abstract}

Day-care center, Permanence and dropout rate

Citación: ANCHONDO-CHAVARRIA, Guadalupe, MARTÍNEZ-ARANDA, Rebeca y PRIETO-GARCÍA, Brenda. Impacto en los niveles de deserción y reprobación en los estudiantes usuarios de la estancia infantil de la Universidad Tecnológica de Chihuahua. Revista de Gestión Universitaria. 2019. 3-7: 9-15

\footnotetext{
*Correspondencia del Autor (correo electrónico: ganchondo@utch.edu.mx)

$\dagger$ Investigador contribuyendo como primer autor.
} 


\section{Introducción}

En el 2012 el Cuerpo Académico Desarrollo de Negocios de la Universidad Tecnológica de Chihuahua detecta un gran número de estudiantes que acuden a clases con sus hijos o que truncan sus estudios por ser padres o madres y propone la creación de una Estancia Infantil con el objetivo de apoyar a los estudiantes padres de familia en el cuidado de sus hijos mientras estudian, se realizan las investigaciones pertinentes y las gestiones para llevar a cabo dicho proyecto el cual inicia operaciones en mayo 2016.

El estudio tiene como objetivo conocer el impacto de la estancia infantil para hijos de estudiantes de la Universidad Tecnológica de Chihuahua en los índices de reprobación, permanencia y deserción a tres años de su fundación. La investigación se desarrolla de la siguiente manera: partiendo del marco teórico que argumenta sobre las razones de realizar la investigación, se describe la metodología, instrumento de recolección de información, muestra y muestreo, para después generar los resultados a partir de los hallazgos y conclusiones del proyecto.

\section{Metodología}

\section{Población y muestra}

Estudiantes de la UTCH padres de familia que hacen uso del servicio de la estancia infantil. De la población anterior se elige trabajar con el $100 \%$ de los estudiantes padres de familia que actualmente utilizan el servicio de la estancia. La investigación se realiza bajo un diseño concluyente, descriptivo y transversal a través de un cuestionario estructurado donde la información se procesa en el software estadístico SPSS. Se analiza el historial académico de los 125 padres de familia que han tenido a sus hijos en la universidad.

\section{Maco Teórico}

\section{Madres adolescentes en México}

El tema de embarazo en las jóvenes se torna como un problema de atención prioritaria para México, ya que el aumento de los adolescentes de 10 a 19 años ha sido constante durante las últimas décadas y se estima que continúe la tendencia durante los próximos años.
Según datos de la COESPO (XXXX), se calcula que para el año 2030, el volumen de población de estas edades en el Estado de México, aumente a 3 millones 74 mil 798 mujeres y hombres.

El crecimiento de la población joven no es el único factor para el índice de embarazos, sin embargo, sí es un punto de referencia que puede potenciar la incidencia de éstos en la población joven; cuando nos referimos al aumento del volumen de mujeres y hombres adolescentes, hablamos del aumento de la población en edad fértil. Los jóvenes inician a tener relaciones sexuales a una edad temprana, ignorando la información que existe para prevenir enfermedades y embarazos no deseados. (COESPO, 2016).

Las campañas informativas de salud de reproducción y los programas sociales se despliegan con gran intensidad por todo el país, sin embargo no alcanzan los resultados deseados por el gobierno. La tasa en niñas adolescentes entre 12 y 19 años ha sido de 79 por cada mil mujeres.

Lorena Ferrer Arreola, jefa de la División de Salud Reproductiva del IMSS, apuntó que según la Encuesta en Salud de 2017, $33.4 \%$ de mujeres adolescentes inició relaciones sexuales sin protección anticonceptiva. Así, la posibilidad de embarazos podría ser de hasta 870 mil. Se registran 16 millones de embarazos no deseados en México ocupando el primer lugar entre las naciones que forman parte de la Organización para la Cooperación y el Desarrollo Económicos (Patricia López 2028).

El embarazo adolescente se cataloga como un problema de salud pública, que trae problemas familiares, escolares y personales, afectando el proyecto de vida de los jóvenes, ya que de dos millones de nacimientos al año en nuestro país, la quinta parte son en mujeres menores de 19 años y 10 mil en madres menores de 14 años. (patricia López 2018)

Según especialistas en el tema, el embarazo en México crecerá si no se toman las medidas necesarias. La ENSAUT (Encuesta Nacional de Salud y Nutrición) anuncia que de no atenderse el tema del embarazo en edad temprana, podría aumentar debido a que no utilizan métodos anticonceptivos o por el desconocimiento que existe del tema. (Romero, 2016).

ANCHONDO-CHAVARRIA, Guadalupe, MARTÍNEZ-ARANDA, Rebeca y PRIETO-GARCÍA, Brenda. Impacto en los niveles de deserción y reprobación en los estudiantes usuarios de la estancia infantil de la Universidad Tecnológica de Chihuahua. Revista de Gestión Universitaria. 2019 
Es importante reconocer esta problemática ya que desencadenan otras situaciones negativas como la misma desintegración familiar, el aislamiento, depresión, y una de las más importantes la necesidad de abandonar su preparación académica, adquiriendo responsabilidades nuevas y complicadas.

En definitiva el embarazo y maternidad en las jóvenes adolescentes es un riesgo que debe prevenir el gobierno, para ello es importante conocer las principales causas por las que una joven se embaraza.

\section{Factores de vulnerabilidad en madres adolescents}

Varias investigaciones proponen descubrir el sentido positivo que la maternidad a edad temprana tiene en el contexto familiar y social. Por ejemplo muchos padres de estas jóvenes piensan que tienen la oportunidad de ir haciendo familia en tiempo y forma, criando a los hijos con mayor fuerza física, sin embargo existen otras investigaciones, dónde se dice lo contrario, que no cuentan con una madurez emocional suficiente y tampoco con la economía necesaria para la manutención de una familia (Claudio Sterrn 2004).

Estudios recientes demuestran que las mujeres adolescentes son cada vez más propensas o vulnerables a los embarazos no deseados, POR lo que deben implementarse cambios estructurales en la sociedad como el que las familias sean más pequeñas, el cambio de pensamiento de querer formar una familia a temprana edad, etc., para que existan cambios positivos en el tema de la maternidad en la adolescencia.

Existen diferentes tipos de vulnerabilidad como el contexto en el que viven los jóvenes, las vivencias, las relaciones sociales y la personalidad del joven. Estos factores son determinantes para generar un buen equilibrio de vida o todo lo contrario. Los especialistas coinciden en que no existe una educación suficiente en el tema de la sexualidad. La información es poca entre la familia y los jóvenes. Las campañas de información tampoco son suficientes, pues siguen los embarazos en adolescentes y embarazos no deseados. La tasa más alta de mujeres embarazadas radica más en las jóvenes que no tienen expectativas educativas respecto a las que sí las tienen.
Se deben reforzar los procesos educativos, impartiendo mejores servicios para los jóvenes en cuanto a los embarazos no deseados, y no sólo información aislada. Deben de crear más prácticas que sean válidas en las escuelas, como en la familia (Binstock \& Näslund, 2013).

Estadísticas de embarazos en adolescentes y jóvenes en Chihuahua muestran que el estado ocupa el segundo lugar a nivel nacional de embarazo adolescente, con 8 mil 738 consultas por primera vez en embarazadas de 15 a 19 años y 429 menores de 15 años. El titular de la Secretaría de Salud Estatal Enrique Grajeda, comenta que de acuerdo a la Organización Mundial de la Salud (OMS, 2009), 49 de cada mil mujeres que dan a luz son adolescentes de 15 a 19 años. De los 1.5 son menores de quince años, el embarazo en la adolescencia es uno de los factores causantes de la muerte. Los nacimientos se incrementaron de 69 a 77 por cada mil mujeres, convirtiendo a Chihuahua en el estado que ocupa el sexto lugar siendo los primeros Coahuila, Durango y Puebla.

Las consecuencias de no llevar a cabo un embarazo bien planeado son las limitaciones de las oportunidades que puedan tener las jóvenes en el ámbito escolar o profesional, tener un ingreso económico bajo, continuar con los estudios y las muertes maternas que se generan especialmente en adolescentes.

En cuanto a los hombres se observa la falta de orientación por parte de las familias, por lo que se quedan solo con lo que se les imparte en las escuelas o centros de salud. Aunque los jóvenes cuenten con información sobre embarazos no deseados sigue existiendo un alto porcentaje de estos. (Instituto Chihuahuense de la Mujer, 2013).

\section{Dificultades que enfrentan estudiantes universitarios padres de familia}

Ser estudiante universitario y a la vez padre o madre de familia, representa una condición de desigualdad e inequidad de género, condición que logran afrontar gracias al apoyo de familiares o instituciones que brindan el servicio de estancia infantil. Los alumnos en esta situación presentan diversas problemáticas que muchas de las veces no les permiten conciliar el rol de padres de familia y el de estudiante. 
Entre las principales dificultades que se presentan se encuentran altos niveles de estrés, al verse obligados a cumplir con tiempos para llevar y recoger a los hijos de la guardería; así como tener que posponer sus estudios por esta causa (Flores, Alejandre, \& Martínez, 2016).

Miller y Arvizu (2016) señalan que las madres estudiantes interrumpen de manera más frecuente los estudios, además buscan actividades económicas o empleos formales para tener ingresos y apoyar al sustento familiar; la mayoría se desempeñan en el comercio informal, en la misma universidad y en sus ratos libres.

Por su parte Hernández (2011) menciona que el embarazo no deseado o a edad temprana trae como consecuencias la interrupción del proyecto educativo de una persona. Por lo cual tienen que dejar sus estudios para atender su embarazo y después a su hijo. Esto trae como consecuencia que se queden sin terminar una carrera profesional, dedicándose solo a ser madre de familia, atendiendo varias actividades que les impiden seguir preparándose.

\section{Implementación del servicio de estancias infantiles en las universidades}

Los alumnos universitarios padres de familia afrontan una serie de dificultades en la crianza de sus hijos, muchos de ellos recurren al apoyo de sus familiares o bien al servicio de estancias infantiles como alternativa que les permita concluir sus estudios. Las guarderías en las universidades han sido un gran apoyo para estos alumnos, logrando reducir el ausentismo y aumentando la motivación para que continúen preparándose profesionalmente, además les brindan mayor tranquilidad al permitirles tenerlos cerca. Estos centros de cuidado diario resuelven un problema social importante, y cuando funcionan en forma óptima, son un apoyo para asegurar el resguardo de los niños; representan también un ahorro económico para los padres de familia. (Hernández, 2013)

Como resultado de esta problemática social varias universidades del país se han enfocado en brindar el servicio de guardería como apoyo para evitar la deserción de los alumnos a causa de este factor. Entre las universidades con servicio de estancia infantil se encuentra la Universidad de Guadalajara, la cual comenzó a brindar el servicio de guardería en agosto de 2012.
Este servicio surge como resultado de un proyecto piloto de la Secretaría de Educación Pública (SEP) de equidad de género, para apoyar a instancias que tienen espacios disponibles para adecuar una estancia infantil. Los resultados obtenidos han sido favorables, por ello se han implementado más estancias infantiles en la Red Universitaria (López, 2016).

Otra Universidad que ofrece el servicio de guardería es la Universidad de Sinaloa, la cual brinda este servicio de manera gratuita a los alumnos que tengan hijos de uno a tres años de edad. Esta estancia surge en octubre de 2012 gracias al apoyo del Programa Integral de Fortalecimiento Institucional (PIFI) y a los recursos propios de esta institución (Universidad Autónoma de Sinaloa, 2012).

De igual manera, la Universidad Autónoma de Nayarit comenzó a ofrecer este servicio de manera gratuita en noviembre de 2016, con la finalidad de reducir los índices de deserción de alumnos con hijos pequeños (Universidad Autónoma de Nayarit, 2016).

En el Estado Chihuahua, la Universidad Autónoma de Ciudad Juárez brinda el servicio estancia infantil a sus alumnos desde agosto de 2008; la estancia infantil otorga la posibilidad a las madres estudiantes de continuar con sus estudios sin descuidar a sus hijos (El Universal, 2008).

\section{Resultados del Estudio de Factibilidad para la apertura de una casa de cuidado diario para estudiantes jefes de familia de la UTCH (Universidad Tecnológica de Chihuahua)}

La población de jóvenes padres de familia en educación superior se incrementa año con año, estos alumnos forman parte de una población que se excluye de la terminación de una profesión o de la obtención de mejores trabajos, esto resulta en una mala calidad de vida por falta de buenas oportunidad laborales.

De acuerdo a los resultados que se obtuvieron de la investigación del Estudio de Factibilidad de la guardería de la UTCH (Cuerpo Académico Desarrollo de Negocios y Consultoría, 2013), se demostró que el apoyo de las familias es imprescindible para poder continuar con los estudios, siendo el $75 \%$ el que menciona que mientras ellos estudian, dejan al hijo con los abuelos o con el padre o madre del hijo.

ANCHONDO-CHAVARRIA, Guadalupe, MARTÍNEZ-ARANDA, Rebeca y PRIETO-GARCÍA, Brenda. Impacto en los niveles de deserción y reprobación en los estudiantes usuarios de la estancia infantil de la Universidad Tecnológica de Chihuahua. Revista de Gestión Universitaria. 2019 
Sin embargo cuando se les presentan asuntos laborales o domésticos, deben tomar la decisión de faltar a clases o se los llevan a la universidad, ocasionando problemas de atención en el aula.

El 58\% de los estudiantes son madres o padres solteros o divorciados, mientras el porcentaje restante son casados o viven en unión libre. Un $60 \%$ se hace cargo totalmente de la manutención de su hijo, por lo que percibe un ingreso de un trabajo de medio o tiempo completo. Estos estudiantes que tienen un empleo formal suelen estar más motivados y comprometidos por terminar sus estudios $\mathrm{y}$ mantener un buen desempeño académico.

El 92\% de los alumnos menciona que sí dejarían a sus hijos en una estancia infantil dentro de la universidad donde estudian, ya que así no tendrían que suspender sus estudios, y así tendrían oportunidad de terminar una carrera, aparte de poder tener un trabajo formal (Anchondo, Valdés y Díaz 2013).

\section{Estudiantes con hijos en la guardería de la UTCH}

El $75 \%$ de estudiantes de la Universidad Tecnológica de Chihuahua tienen hijos entre uno y seis años de edad por lo que para poder asistir a clases, los tienen que dejar al cuidado de familiares o guarderías privadas.

La universidad adaptó una casa de cuidado diario para cubrir las necesidades de estudiantes durante la jornada académica, lo que les permite continuar y terminar con sus estudios. Actualmente atiende a un total de 35 niños y niñas. Gracias a un convenio de colaboración con Casas de Cuidado Diario, la universidad ofrece ese servicio en su plantel, para brindar el apoyo exclusivo a estudiantes que son madres o padres de familia.

De acuerdo a un estudio realizado por la institución, alrededor del 75 por ciento de las y los estudiantes, son padres o madres de familia, y para acudir a sus clases, dejan a las y los menores con familiares o en guarderías privadas. Ante esta situación, se estableció dicha alianza estratégica y hasta el momento, un total de 35 niños y niñas de entre uno y seis años de edad, son atendidos por un grupo de especialistas, en un área que fue adecuada en la propia universidad, lo que brinda mayor seguridad y cercanía a las y los usuarios.
Las Casas de Cuidado Diario son un modelo alternativo para la atención y protección de niñas y niños pequeños, hijos de madres trabajadoras que no cuentan con un servicio adecuado para dejarlos en lo que realizan su jornada laboral, por lo que en este caso la UTCH adaptó este sistema para cubrir las necesidades de las y los estudiantes con hijas e hijos pequeños, con la finalidad de que puedan dejarlos al cuidado de un equipo profesional y confiable, durante su jornada académica diaria, lo que permite que puedan continuar y concluir sus estudios. (León, 2019).

\section{Resultados}

La Estancia actualmente atiende a 36 niños de los cuales 12 son del turno matutino y 24 del turno despresurizado o nocturno. Desde que se fundó se han atendido 56 niños en el turno matutino y 69 en el despresurizado.

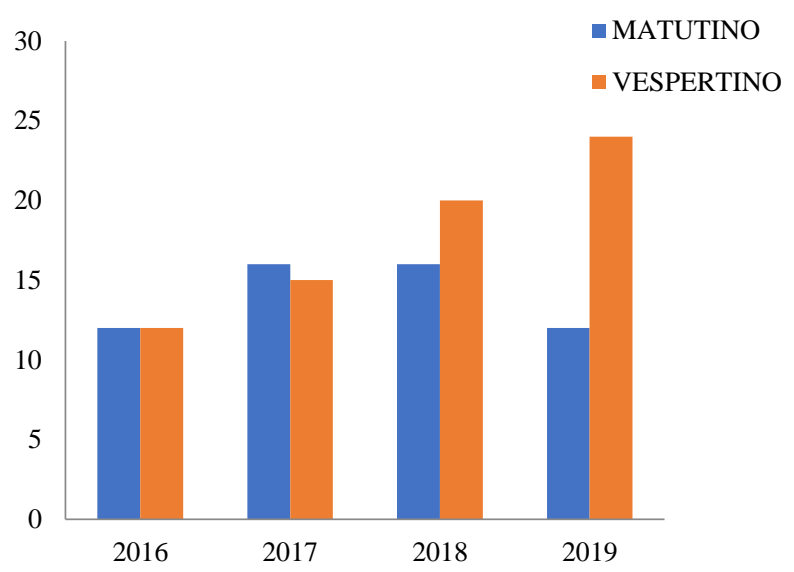

Gráfico 1 Niños Inscritos en Estancia Infantil de la UTCH Fuente: Elaboaración Propia

El 69.4 de los estudiantes tienen hijo único y el $16.7 \%$ dos hijos. Una de las razones por las cuales hay más niños en el turno despresurizado es porque la Estancia aún no cuenta con el servicio de kínder y cuando el niño cumple la edad para asistir a este, deja la guardería. Existe la propuesta por parte del cuerpo académico de la compra de un vehículo que permita trasladar a los niños a un kínder cercano a la zona y así ampliar el número de niños en este turno.

Las carreras que más utilizan el servicio son: Procesos de Producción con un 49\%, seguido de Desarrollo de Negocios con un 35\%. 


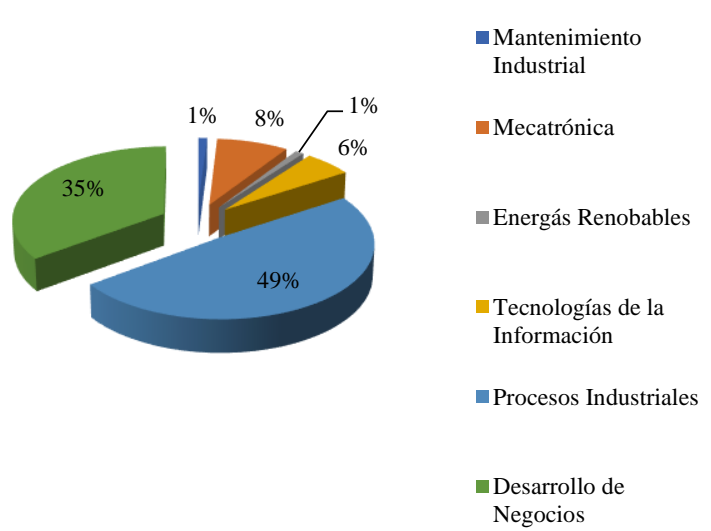

Gráfico 2 Uso Estancia Infantil por Carrera Fuente: Elaboaración Propia

El $72.2 \%$ menciona que factor decisivo para ingresar a la universidad, es que esta brinde el servicio de guardería. Un $83 \%$ de los alumnos entrevistados comentan que su promedio global se incrementó a raíz de tener un lugar cercano y de calidad donde tener a sus hijos.

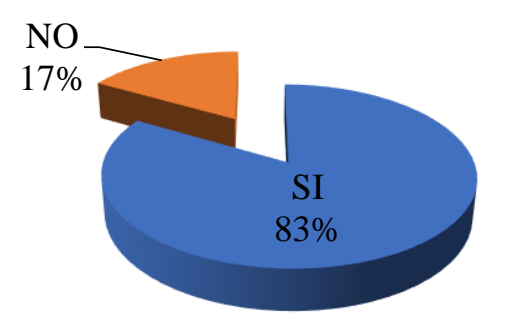

Gráfico 3 Porcentaje de Alumnos que han mejorado sus calificaciones

Fuente: Elaboaración Propia

El 97\% comenta que este servicio ha contribuido a su permanencia en la universidad. Los resultados obtenidos en la investigación señalan que la estancia infantil ha sido benéfica y de gran apoyo para el desarrollo de los padres y madres de familia que hacen uso del servicio, consideran que han incrementado su nivel académico, ha disminuido la reprobación ya que han bajado su nivel de estrés al tener un horario cómodo, al no tener que trasladarse a otro lugar lejos de la universidad para dejar a sus hijos y también ha tenido un beneficio económico ya que cuentan con un programa de pago tripartita: una parte la paga la universidad, otra Casas de Cuidado Diario A.C. y la otra el estudiante.

El 100\% de los estudiantes están muy satisfechos del servicio que ofrece la estancia y la única sugerencia que hay es ampliar la edad de los niños que se aceptan. Gráfica elaboración propia
En el estudio del historial académico de los 125 alumnos padres de familia encontramos que de los 25 hombres solo reprobaron 3 y de las mujeres 4.

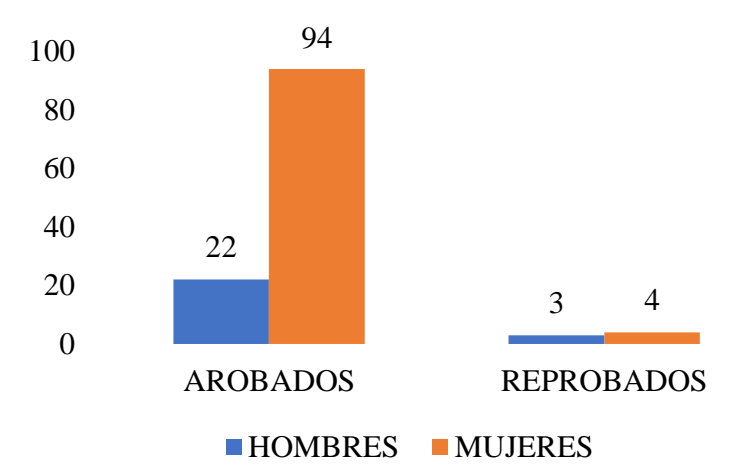

Gráfico 4 Historial académico de alumnos con hijos en la estancia

Fuente: Elaboaración Propia

Del total de alumnos que no concluyeron sus estudios son 4 hombres y 2 mujeres los cuales señalan que la causa de deserción es por cuestiones económicas y cambios en turnos del trabajo y las mujeres por cuestiones familiares.

\section{Conclusiones}

La Universidad, se considera una extensión de la familia, como parte de un grupo normativo en donde parte de las responsabilidades es desarrollar personas con valores éticos y morales en beneficio de la sociedad. El impacto y la responsabilidad social se traduce en ampliar las posibilidades de incorporar jóvenes a los estudios universitarios para que logren obtener un mejor trabajo y poder en un futuro ser parte de la estructuración y formación de un país con mayores esperanzas de progreso y estabilidad social.

El contar con un servicio de guardería ha ayudado a los jóvenes en su desarrollo personal y profesional, reduciendo la desigualdad de oportunidades derivadas de su condición de padre y madre y a la vez estudiante universitario. La estancia infantil ha disminuido la deserción escolar y ha mejorado el rendimiento académico de estos jóvenes, lo cual se traduce en mejores calificaciones. Este servicio ha beneficiado a las familias al fortalecer su integración y su entorno social, generando con esto un efecto multiplicador. 
El servicio de estancia infantil ofrecido por la UTCH ha hecho frente a una problemática social latente y ha permitido que los alumnos en esta condición puedan concluir sus estudios, brindándoles la posibilidad de obtener un mejor empleo y con ello mejorar la calidad de vida de su familia.

\section{Referencias}

Alicia Xochitl Olvera Rosas. (Segunda edición 2014). Embarazo adolescente y madres jóvenes en México. Una visión desde el Promajoven, 3, 180.

Anchondo, Valdés y Díaz. (2013). Apertura de una estancia infantil para hijos de estudiantes de la UTCH y su impacto. 2 de Septiembre del 2019, de CIPITECH Sitio web: https://es.scribd.com/document/318593402/ME MORIA-SEXTO-CONGRESOINTERNACIONAL-DE-INVESTIGACIO-NCIPITECH-2013-UTCH-1.

Claudio Sterrn. (ene/marzo 2004). Vulnerabilidad social y embarazo adolescente en México. Colegio de México, 10 No. 39, 30.

COESPO (2016). Embarazo adolescente. 26 de agosto 2019, de COESPO Sitio web: www.edomex.gob.mx/coespo (Consejo Estatal de Población).

Eduardo León. (25 de Marzo del 2019). UTCH ofrece guardería. 20 de agosto del 2019, de http://www.omnia.com.mx/noticia/102599/utch -ofrece-casa-de-cuidado-diario-a-sus-alumnosque-son-padres-de-familia Sitio web: http://www.omnia.com.mx/noticia/102599/utch -ofrece-casa-de-cuidado-diario-a-sus-alumnosque-son-padres-de-familia.

Georgina Binstock y Emma Näslund-Hadley. (oct./dic. 2013). Maternidad adolescente y su impacto sobre las trayectorias educativas y laborales de mujeres de sectores populares urbanos. Papeles de población, Pap. Poblac. vol.19 no.78, 1-30.
Instituto Chihuahuense de la Mujer. (2013). DIAGNOSTICO PROSPECTIVO PARA DETECTAR LAS CAUSAS DEL EMBARAZO EN ADOLECENTES EN LOS MUNICIPIOS DE CHIHUAHUA Y JUARÉZ. 21 de Agosto del 2019, de Instituto Chihuahuense de la Mujer Sitio web: cedoc.inmujeres.gob.mx/.../Chihuahua/CHI_M A18_dx_prospectivo_2012.

Katherine Hernández Pérez* Eilyn Orozco Romero*. (2011). Embarazo en estudiantes de carreras universitarias. 5 de Septiembre del 2019, de Dictamen Libre, 8 Sitio web: https://www.google.com/search? source=hp\&ei $=$ AjtxXfbREoiStQWe27DgBQ\&q=embarazo+e $\mathrm{n}+$ estudiantes+de+carreras+universitarias\&oq= Embarazo+en+estudiantes+de+carreras+univer sitarias\&gs_l=psyab.1.0.0.7813.7813..10083...0.0..0.116.116.0j1.. ....0....2j1..gws-wiz.a0Tsm7QqFd4

Laura Romero. (29 de septiembre de 2016). Embarazo adolescente problema de salud pública. Gaceta UNA0M, 3, 4-

Patricia Lopez. (29 de Septiembre del 2018). Embarazo adolescente problema de salud pública. GACETA UNAM, 4,816, 35. 